\title{
Infection of Plasmodium falciparum and helminths among school children in communities in Southern and Northern Ghana
}

\author{
G. Akosah-Brempong, S. K. Attah, I. A. Hinne, A. Abdulai, K. Addo-Osafo, E. L. Appiah, M.-M. Osei and \\ Y. A. Afrane*
}

\begin{abstract}
Background: Infections of Plasmodium species, Schistosoma species and soil-transmitted helminths (STH) inflict a significant burden on children mostly in deprived communities in Ghana. Despite the deployment of malaria vector control and the annual Mass Drug Administration by National Control Programmes, these infections still pose major public health concerns in Ghana. Some remote communities which are hard-to-reach are not covered by MDA campaigns which is a major challenge to meeting elimination targets. Adequate data is necessary for formulating policies and strengthening interventions to mitigate transmission. This study assessed the infection burden of Plasmodium, Schistosoma species and STH infections among school children in communities in Southern and Northern Ghana.

Method: School children living in communities in Southern (Ada Foah, Pediatorkope, Tuanikope) and Northern (Kpalsogu) Ghana were enrolled in a cross-sectional study. A total of 493 (241 males and 252 females) school children aged (2-15 years) were enrolled in the study. Stool samples were collected to screen for Schistosoma mansoni and STH infections using the formol-ether concentration technique and urine samples were also collected to screen for $S$. haematobium using the routine urine examination method. Plasmodium parasitaemia was determined from thick and thin finger-prick blood samples.

Results: Overall, the prevalence of P. falciparum, S. mansoni, S. haematobium, Trichuris trichiura and hookworm infections were $17.2 \%(95 \% \mathrm{Cl} 12.8-19.7), 22.6 \%(95 \% \mathrm{Cl} 25.2-32.7), 1.6 \%(95 \% \mathrm{Cl} 0.89-5.2), 1.2 \%(95 \% \mathrm{Cl} 0.78-4.8)$ and $1.2 \%$ (95\% Cl 0.78-4.8) respectively. Plasmodium falciparum infection was generally widespread in all the study sites with Ada Foah recording the highest prevalence (35.3\%) and Kpalsogu recording the lowest (5.8\%). Schistosoma mansoni was present in only two Southern communities with Tuanikope recording the highest prevalence of $70.3 \%$ as against $51.5 \%$ recorded in Pediatorkope. A total of $4.5 \%(95 \% \mathrm{Cl} 2.82-10.8)$ of the children were co-infected with P. falciparum, Schistosoma species and STHs. This occurred only in the Southern communities; of which combination of P. falciparum and S. mansoni were predominant (1.4\%).
\end{abstract}

Conclusion: A relatively low burden of parasites co-infection among children only in the Southern communities was detected. However, there were a high prevalence of single infections of P. falciparum and S. mansoni in those

\footnotetext{
*Correspondence: yaw_afrane@yahoo.com

Department of Medical Microbiology, University of Ghana Medical

School, College of Health Sciences, University of Ghana, Korle-Bu, Accra,

Ghana
}

(C) The Author(s) 2021. Open Access This article is licensed under a Creative Commons Attribution 4.0 International License, which permits use, sharing, adaptation, distribution and reproduction in any medium or format, as long as you give appropriate credit to the original author(s) and the source, provide a link to the Creative Commons licence, and indicate if changes were made. The images or other third party material in this article are included in the article's Creative Commons licence, unless indicated otherwise in a credit line to the material. If material is not included in the article's Creative Commons licence and your intended use is not permitted by statutory regulation or exceeds the permitted use, you will need to obtain permission directly from the copyright holder. To view a copy of this licence, visit http://creativecommons.org/licenses/by/4.0/. The Creative Commons Public Domain Dedication waiver (http://creativeco mmons.org/publicdomain/zero/1.0/) applies to the data made available in this article, unless otherwise stated in a credit line to the data. 
communities. Control measures for the helminths needs to be restarted in the island communities with a high burden of S. mansoni infections and that of Plasmodium needs to be scaled up in Ada Foah where P. falciparum infections were high.

Keywords: Plasmodium falciparum, Schistosoma mansoni, Schistosoma haematobium, Soil-transmitted helminths, Ghana

\section{Background}

Infections of Plasmodium species, Schistosoma species and soil-transmitted helminths (STHs) are the leading parasitic infections with a considerable disease burden in pre-school and school-going children particularly in Sub-Saharan Africa [1-3]. Plasmodium infection and Neglected Tropical Diseases (NTDs) are reported to affect millions of lives in developing countries owing to their great association with poor sanitary conditions, unsafe water sources for domestic activities and low socio-economic status $[4,5]$. The distribution of these diseases often overlaps due to various conditions that favour multiple parasitic species survival and transmission [6].

Malaria caused by the Plasmodium parasites is endemic and perennial in all parts of Ghana with seasonal variations more pronounced in the northern part of the country. Plasmodium falciparum accounts for over $95 \%$ of malaria infections of about 4.5 million morbidity $[7,8]$.

Ghana is among the most schistosomiasis-burdened countries in sub-Saharan Africa with two main human species, Schistosoma mansoni and S. haematobium causing the disease in various parts of the country where there are freshwater bodies that support the snail vectors [9]. In some communities along the Volta basin in Southern Ghana, prevalence of $S$. mansoni is reported to be as high as $80-90 \%$ [10]. A systematic review showing the prevalence, types, geographical and population distribution of soil-transmitted helminths in Ghana over a 10-year period of 2000-2018, identified hookworms, Ascaris lumbricoides and Trichuris trichiura as the most widespread STHs infections [11]. These infections affect most people living in poor sanitary areas where children, pregnant women and farmers are vulnerable to these infections. The prevalence of STHs in most endemic communities in Ghana has reduced significantly over the years since the scale up of MDA campaigns in some areas [10].

Children co-infected with these parasites develop below optimal and they have increased susceptibility to other infections. They have reduced learning ability which results in poor school achievements $[12,13]$. Poly-parasitism acts in multiple ways to adversely affect human health through immune suppression, nutrient malabsorption, anorexia, and haemolysis.
In Ghana, these infections are a major health problem among school children due to their exposure to these parasites in their daily activities which leads to poor cognitive development, retarded growth, general ill health and eventually death $[14,15]$.

Co-infections of P. falciparum, Schistosoma species and STHs have been reported from various epidemiological settings in Africa [16]. However, most of these parasitic infections are studied individually thus, data on prevalence, morbidity and mortality associated with multiple parasitic infections in Ghana are limited. More so, some communities in Ghana that are hard-to-reach such as the island communities are not covered by MDA campaigns which is a major challenge to meeting elimination targets.

Knowledge on the extent of parasite co-infections in high-risk groups will serve as a guide in developing sound integrated intervention strategies to reduce the burden of these diseases and co-morbidity. This study was therefore conducted to assess the infection burden of Plasmodium, Schistosoma species and STH among school children living in communities in Southern and Northern Ghana.

\section{Materials and methods \\ Study communities}

The study was carried out in three (3) Southern communities (Tuanikope, Pediatorkope and Ada Foah) all in the Ada East District of the Greater Accra region of Ghana and one (1) Northern community (Kpalsogu) in the Kumbugu District of the Northern region of Ghana (Fig. 1).

Tuanikope $\left(5^{\circ} 48^{\prime} \mathrm{N}, 0^{\circ} 37^{\prime} \mathrm{E}, 3 \mathrm{~m}\right.$ above sea level) and Pediatorkope $\left(5^{\circ} 49^{\prime} \mathrm{N}, 0^{\circ} 37^{\prime} \mathrm{E}, 3 \mathrm{~m}\right)$ are island communities on the Volta River. The inhabitants are mainly engaged in fishing and rural subsistence farming. Malaria transmission occurs all year round with seasonal peaks reaching high in the rainy season as habitats for vectors increases. These rural communities lack basic social amenities and access to potable water, and thus, depend on river water for domestic chores increasing the risk of helminths infections. These two island communities lie in the coastal savannah ecological zone in Ghana which has a bimodal rainfall pattern. The main rainy season is from April to June and the main dry season is from January to March. 


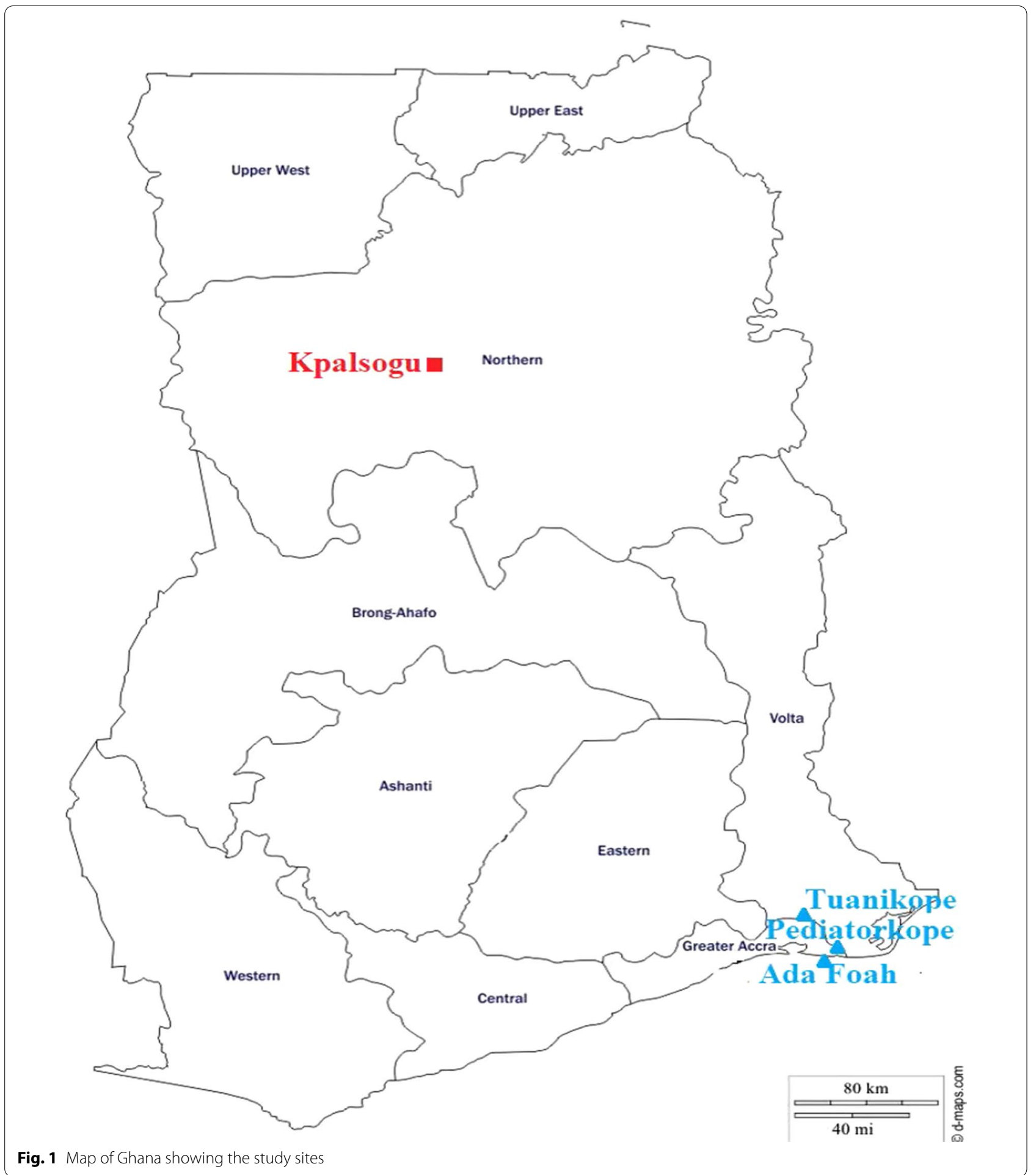

Ada Foah $\left(5^{\circ} 45^{\prime} \mathrm{S}, 6^{\circ} 00^{\prime} \mathrm{N}, 3 \mathrm{~m}\right.$ above sea level) is a coastal community that borders the Volta River to the east. The majority of the inhabitants are engaged mainly in fishing and vegetable farming. Malaria transmission occurs throughout the year as ponds and wells in vegetable farms sustain the breeding of vectors. Intense malaria transmission occurs in the rainy season (March-November). Ada Foah is also found in the coastal savannah zone of Ghana. 
Kpalsogu $\left(9^{\circ} 34^{\prime} \mathrm{N}, 1^{\circ} 01^{\prime} \mathrm{W}, 124 \mathrm{~m}\right.$ above sea level) is a farming community in the Kumbungu district in the Northern region of Ghana which lies within the Sahel savannah ecological zone in Ghana with a unimodal rainfall pattern. The rainy season is from May to November and the dry season from December to April. The community has a Dam used for irrigational farming and domestic purposes and also lacks basic amenities which contributes to malaria and helminths transmission.

Each of the four (4) communities has only one basic school which was chosen for the study. The study was carried out between April and May 2019 among 493 school children from four (4) primary schools. Every primary school child who was willing to be part of the study and whose parents provided informed consent was considered for the study.

\section{Study design and population}

A cross-sectional study was undertaken to determine the prevalence and intensity of Plasmodium parasites, $S$. mansoni, S. haematobium and STHs infections among school children in the study sites. Demographic data including the age and gender of the study participants were recorded.

\section{Recruitment and sample collection}

All primary school-going children aged 3-15 years, in each of the study communities were requested to be part of the study after explaining the purpose of the study to them and their parents/guardians. Those who consented were recruited from each class and signed an assent form with their parents. Each child was screened for infection with Schistosoma (stool and urine), STHs (stool) and Plasmodium (blood).

\section{Stool collection and parasite determination.}

Fresh stool samples in containers labelled with unique identification codes were collected from the school children in May, 2019. The stool samples were taken to the Parasitology Laboratory of the Medical Microbiology Department, University of Ghana Medical School. The formol-ether concentration technique was used to analyze the samples. Briefly, one (1) gram of each stool sample was emulsified in $10 \mathrm{ml}$ of $10 \%$ formalin solution in a centrifuge tube using an applicator stick. The suspension was then strained through two layers of gauze into another centrifuge tube. More of the $10 \%$ formalin solution was added to the strained suspension to bring the volume to $10 \mathrm{ml}$. Three (3) millilitres of ether was then added to the suspension, the tube vigorously shaken for $10 \mathrm{~s}$ and the sample centrifuged for $3 \mathrm{~min}$ at $1000 \mathrm{rpm}$. The ether-faecal debris and formalin were decanted in a single movement. The sediment was deposited on a clean slide and examined microscopically for schistosome and STH parasite eggs. Infection intensities were classified into light, moderate, and heavy egg counts of 1-99 epg (egg per gram), 100-399 epg and > 400 epg respectively for S. mansoni, and 1-999 epg, 1000-9999 epg and $>10,000$ epg respectively for STHs [16].

\section{Urine collection and parasite determination}

Each of the study participants was given a labelled $50 \mathrm{ml}$ wide-mouth screw cap container to provide, at least, $15 \mathrm{ml}$ of urine on the day of screening. About $1 \mathrm{ml}$ of $10 \%$ formalin was added to each urine sample and transported to the Parasitology Laboratory of the Medical Microbiology Department, University of Ghana Medical School for examination.

Each urine sample was homogenized, and $10 \mathrm{ml}$ transferred into an unused $15 \mathrm{ml}$ centrifuge tube. The samples were centrifuged at $\times 1000 \mathrm{rpm}$ for $5 \mathrm{~min}$, the supernatant carefully decanted, and the deposits examined for schistosome eggs using the $\times 10$ objectives of the light microscope. The intensity of infection was classified as light when the egg count was less than 50 eggs/10 $\mathrm{ml}$ of urine and heavy when egg counts are more than 50 eggs per $10 \mathrm{ml}$.

\section{Screening of school children for malaria parasites}

Finger prick blood samples were collected after cleaning the finger surface using an alcohol swab. Thick and thin blood smears were prepared with the latter fixed with methanol and stained with $10 \%$ Giemsa. Plasmodium parasite densities were estimated from thick smears under the light microscope using the $\times 100$ objective to count the number of parasites per 200 white blood cells (WBC) from the thick smears. The counts of $P$. falciparum were converted to the number of parasites per $\mu \mathrm{l}$ of blood, assuming a standard WBC count of $8000 / \mu \mathrm{l}$. Thin blood smears were examined for Plasmodium species identification.

\section{Data analysis}

Data were entered into Microsoft Excel 2013 spreadsheets, checked for entry errors, and analyzed using STATA version 15 software (StataCorp. 2017. Stata Statistical Software: Release 15. College Station, TX: StataCorp LLC). The prevalence of single and multiple species of parasites was assessed and classified according to gender and age groups. The Fisher's exact test was used to assess differences in infection rates of single and double or triple parasitic infections according to sex, age and study community. Logistic regression analysis was applied to investigate whether gender and age were significantly associated with the $S$. mansoni, $P$. falciparum and hookworm infections. 


\section{Results}

\section{Background characteristics}

A total of 493 primary school children aged 2-15 years were recruited in this study. Slightly more than half (51.12\%) of the participants were females with the majority $(47.67 \%)$ falling within the $6-10$ years age group. The community, age and sex distribution of the study participants are as shown in Table 1.

\section{Prevalence and coinfections pattern of parasites}

Overall, 188/493 (38.1\%, 95\% CI 41.2-47.4) harboured at least one parasitic infection.

Table 2 shows a multivariate analysis of the prevalence pattern of P. falciparum, S. mansoni and S. haematobium. Out of the 493 children screened for malaria parasites, 85

Table 1 Demographic characteristics of study participants

\begin{tabular}{lll}
\hline Background characteristics & Frequency $(\mathbf{n})$ & Percentage (\%) \\
\hline Schools & & \\
Ada Foah R/C & 184 & 37.32 \\
Tuanikope D/A & 64 & 12.98 \\
Pediatorkope D/A & 125 & 25.35 \\
Kpalsogu Zion & 120 & 24.35 \\
Sexes & & \\
Male & 241 & 48.88 \\
Female & 252 & 51.12 \\
Ages & & \\
$2-5$ & 86 & 17.44 \\
$6-10$ & 236 & 47.67 \\
$11-15$ & 171 & 34.89 \\
\hline
\end{tabular}

(17.2\%, 95\% CI 12.8-19.7) were positive for P. falciparum (none of the children harboured other Plasmodium species). Prevalence was significantly high in children from Ada Foah 65 (35.3\%, $p<0.001,95 \%$ CI 8.25-14.7) than those from other communities with Kpalsogu recording the least 7 (5.8\%).

There was a significant difference observed in the prevalence of $P$. falciparum according to age groups $(p=0.04$, $95 \%$ CI $0.07-1.26)$ with the highest $41(24.0 \%)$ recorded in the older age group of 11-15 years followed by age group 6-10 years $37(15.7 \%)$ and lowest in age group $2-5$ years 7 (8.1\%). P. falciparum prevalence showed no significant difference according to gender $(p=0.072)$ although females recorded a higher prevalence of 51 (20.2\%).

The overall prevalence of S. mansoni in the study was 97 (22.6\%, 95\% CI 25.2-32.7). Schistosoma mansoni was present in only two communities with Tuanikope recording a higher prevalence $45(70.3 \%)$ as compared to Pediatorkope 52 (51.5\%). A significant difference in the prevalence of $S$. mansoni was observed between the study communities ( $\mathrm{p}<0.001,95 \%$ CI 25.2-32.7). Males were more infected 54 (23.1\%), than females 43 (18.2\%) and a high proportion of infection rate was observed according to age groups. However, no significant difference was observed according to gender and age (Table 2).

The majority of the children infected with $S$. mansoni (89\%) had light infections (1-100 epg).

The prevalence of $S$. haematobium in the communities ranged from 0 to $2.7 \%$. Out of the 493 participants, $8(1.6 \%$, 95\% CI $0.89-5.2)$ were positive for $S$. haematobium. The observed difference in the prevalence between the study communities was not statistically significant. Infection with $S$. haematobium was

Table 2 A multivariate analysis showing the prevalence pattern of P. falciparum, S. mansoni and S. haematobium

\begin{tabular}{|c|c|c|c|c|c|c|c|c|c|c|c|c|c|}
\hline & \multirow[t]{2}{*}{$\mathbf{N}$} & \multicolumn{4}{|c|}{ P. falciparum infection } & \multicolumn{4}{|c|}{ S. mansoni infection } & \multicolumn{4}{|c|}{ S. haematobium infection } \\
\hline & & n (\%) & aOR & $95 \% \mathrm{Cl}$ & $p$ & n (\%) & aOR & $95 \% \mathrm{Cl}$ & $p$ & n (\%) & aOR & $95 \% \mathrm{Cl}$ & $p$ \\
\hline Total & 493 & $85(17.2)$ & & $12.8-19.7$ & & $97(22.6)$ & & $25.2-32.7$ & & $8(1.6)$ & & $0.89-5.21$ & \\
\hline \multicolumn{14}{|l|}{ Sites } \\
\hline Ada Foah & 184 & $65(35.3)$ & & & $<0.001$ & $0(0)$ & & & $<0.001$ & $5(2.7)$ & & & 0.197 \\
\hline Pediatorkope & 125 & $9(7.2)$ & & $8.25-14.7$ & & $52(51.5)$ & & & & $3(2.4)$ & & & \\
\hline Tuanikope & 64 & $4(6.3)$ & & & & $45(70.3)$ & & & & $0(0)$ & & & \\
\hline Kpalsogu & 120 & $7(5.8)$ & & & & $0(0)$ & & & & $0(0)$ & & & \\
\hline \multicolumn{14}{|l|}{ Sex } \\
\hline Male & 241 & $34(14.1)$ & 1.41 & $0.86-2.30$ & 0.072 & $54(23.1)$ & 0.74 & $0.47-1.18$ & 0.185 & 4(1.6) & 1.28 & $0.25-6.53$ & 1.000 \\
\hline Female & 252 & $51(20.2)$ & & & & $43(18.2)$ & & & & $4(1.7)$ & & & \\
\hline \multicolumn{14}{|l|}{ Age } \\
\hline$>6$ & 86 & $7(8.1)$ & 1.16 & $1.07-1.26$ & 0.004 & $11(12.9)$ & 1.10 & $1.02-1.19$ & 0.095 & $0(0)$ & 1.39 & $1.01-1.91$ & 0.504 \\
\hline $6-10$ & 236 & $37(15.7)$ & & & & $48(20.8)$ & & & & $4(1.7)$ & & & \\
\hline $11-15$ & 171 & $41(24.0)$ & & & & $38(24.8)$ & & & & $4(2.3)$ & & & \\
\hline
\end{tabular}

$P=p$-value $(p<0.05$ is statistically significant, $\mathrm{p}>0.05$ is statistically not significant) 
not gender or age dependent. However, there was an increasing trend with increasing age, with the highest prevalence of $2.3 \%$ recorded for the age group 11-15 years (Table 2). The egg count of participants ranged from 2 to $106 \mathrm{eggs} / 10 \mathrm{ml}$ of urine with only 1 child harbouring a heavy infection.

Hookworm and T. trichiura were the only STHs detected albeit with low prevalence (Table 3). The overall hookworm prevalence which was $1.2 \%$ (95\% CI 0.78-4.8) was recorded only in Pediatorkope and Tuanikope with no significant differences according to the site, gender, and age Prevalence of T. trichiura was also 1.2\% (95\% CI $0.78-4.8$ ) and this was recorded only in Ada Foah (Table 3).
Co-infections in the study participants was low. The prevalence of at least two parasite species in the study participants put together was $22(4.5 \%, 95 \%$ CI $2.82-$ 10.8) (Table 4). Most of these parasite co-infections predominantly occurred in children from Pediatorkope $59 \%(13 / 22)$, where there was no MDA at time of the sample collection. However, with Kpalsogu, where there was ongoing MDA, no co-infections was recorded. Table 4 shows the prevalence of co-infections, stratified by communities, sex and age among the 493 primary schoolchildren. The co-infections between $S$. mansoni and $P$. falciparum 6 (1.4\%) was the most common, followed by co-infections of $S$. haematobium and $P$. falciparum $4(0.8 \%)$. The age group, $6-10$ years showed the

Table 3 A multivariate analysis showing the prevalence pattern of Hookworm and Trichuris trichiura

\begin{tabular}{|c|c|c|c|c|c|c|c|c|c|}
\hline & \multirow[t]{2}{*}{$\mathbf{N}$} & \multicolumn{4}{|c|}{ Hookworm infection } & \multicolumn{4}{|c|}{ Trichuris trichiura infection } \\
\hline & & n (\%) & $\mathrm{aOR}$ & $95 \% \mathrm{Cl}$ & $p$ & n (\%) & $\mathrm{aOR}$ & $95 \% \mathrm{Cl}$ & $p$ \\
\hline Total & 493 & $5(1.2)$ & & $0.78-4.8$ & & $5(1.2)$ & & $0.78-4.8$ & \\
\hline \multicolumn{10}{|l|}{ Site } \\
\hline Ada Foah & 184 & $0(0)$ & & & 0.24 & $5(2.7)$ & & & 0.09 \\
\hline Pediatorkope & 125 & $3(2.4)$ & & & & $0(0)$ & & & \\
\hline Tuanikope & 64 & $2(3.1)$ & & & & $0(0)$ & & & \\
\hline Kpalsogu & 120 & $0(0)$ & & & & $0(0)$ & & & \\
\hline \multicolumn{10}{|l|}{ Sex } \\
\hline Male & 241 & $3(1.2)$ & 0.92 & $0.13-6.36$ & 0.64 & $2(0.8)$ & 0.92 & $0.13-6.36$ & 1.00 \\
\hline Female & 252 & $2(0.7)$ & & & & $3(1.2)$ & & & \\
\hline \multicolumn{10}{|l|}{ Age } \\
\hline$>6$ & 86 & $2(2.3)$ & 0.82 & $0.59-1.14$ & 0.58 & $0(0)$ & 0.74 & $0.67-1.84$ & 0.72 \\
\hline $6-10$ & 236 & $1(0.4)$ & & & & $3(1.3)$ & & & \\
\hline $11-15$ & 171 & $2(1.2)$ & & & & $2(0.85)$ & & & \\
\hline
\end{tabular}

Table 4 Prevalence of co-infections, stratified by communities, sex and age among 493 primary schoolchildren

\begin{tabular}{|c|c|c|c|c|c|c|c|c|c|}
\hline \multirow[t]{2}{*}{ Variable } & \multirow[t]{2}{*}{$N$} & \multicolumn{2}{|c|}{ P. falciparum + S. mansoni } & \multicolumn{2}{|c|}{$\begin{array}{l}\text { P. falciparum }+S . \\
\text { haematobium }\end{array}$} & \multicolumn{2}{|c|}{ P. falciparum $+T$. trichiura } & \multicolumn{2}{|c|}{$\begin{array}{l}\text { S. mansoni }+S \text {. } \\
\text { haematobium }\end{array}$} \\
\hline & & n (\%) & $p(95 \% \mathrm{Cl})$ & n (\%) & $p(95 \% \mathrm{Cl})$ & n (\%) & $p(95 \% \mathrm{Cl})$ & n (\%) & $p(95 \% \mathrm{Cl})$ \\
\hline \multicolumn{10}{|l|}{ Site } \\
\hline Ada Foah & 184 & $5(2.7)$ & $0.94(0.72-1.41)$ & $3(1.6)$ & $0.54(1.42-2.34)$ & $2(1.09)$ & $0.09(0.56-1.93)$ & $0(0)$ & $0.09(0.86-2.04)$ \\
\hline Pediatorkope & 125 & $0(0)$ & & $1(0.9)$ & & $0(0)$ & & $3(2.9)$ & \\
\hline Tuanikope & 64 & $1(1.6)$ & & $0(0)$ & & $0(0)$ & & $0(0)$ & \\
\hline \multicolumn{10}{|l|}{ Sex } \\
\hline Male & 241 & $4(1.9)$ & $0.48(1.14-2.1)$ & $1(0.4)$ & $0.23(1.42-2.34)$ & $0(0)$ & $0.09(0.56-1.93)$ & $3(1.2)$ & $0.08(1.24-3.36)$ \\
\hline Female & 252 & $2(0.9)$ & & $3(1.2)$ & & $2(0.9)$ & & $0(0)$ & \\
\hline \multicolumn{10}{|l|}{ Age } \\
\hline$<6$ & 86 & $1(1.6)$ & $0.78(0.83-3.52)$ & $0(0)$ & $0.16(0.86-2.81)$ & $0(0)$ & $0.10(1.12-2.36)$ & $0(0)$ & $0.09(1.16-2.86)$ \\
\hline $6-10$ & 236 & $2(0.9)$ & & $2(0.9)$ & & $2(0.9)$ & & $2(0.9)$ & \\
\hline $11-15$ & 171 & $3(1.7)$ & & $2(0.9)$ & & $0(0)$ & & $1(0.6)$ & \\
\hline
\end{tabular}


highest prevalence of co-infection 8 (3.4\%) and males also showed a higher prevalence of co-infection.

The prevalence of co-infections did not differ significantly according to age and gender.

\section{Discussion}

The burden of malaria, schistosomiasis, and STH infections particularly among children is a major public health problem in Ghana where co-infections in a single host do sometimes occur. Even though these infections coexist, most studies in Ghana focus on individual infections hence data on the co-morbidity of these infections remains limited. Understanding the epidemiology of these infections and their co-infections is an important finding to support the design of integrated disease control strategies. The results from the study demonstrated that $P$. falciparum, S. mansoni, S. haematobium and STH infections (Hookworm and T. trichiura) are prevalent in school children in communities in Southern and Northern Ghana and co-infections of these parasites were detected at a relatively low rate.

It was found in the study that $P$. falciparum infections were present in school children from all study communities and were highest among children in Ada Foah and infection burden lowest in Kpalsogou. Kpalsogou has been under Indoor Residual Spraying (IRS) implementation since 2008 and together with the Long-Lasting Insecticide Nets (LLINs) coverage of $>70 \%$ in the area, has contributed to reduction in the burden of malaria in the community. There has been no IRS implementation in Ada Foah and the two island sites of Pediatorkope and Tuanikope. This highlights the gains from the current vector control interventions which needs to be sustained and probably expanded to other endemic areas to further reduce malaria burden. The total prevalence of $P$. falciparum infection in school children in this study was moderate which corroborates with infection rates reported in other studies among school children in Southern Ghana [13]. Logistic regression analysis showed an increased malaria risk for older children which was unexpected as infection burden is expected to decrease with increasing age when children gradually develop some degree of immunity against the Plasmodium parasite due to repeated infections. A similar observation was reported in a study in the Democratic Republic of Congo which found a comparably high $P$. falciparum infection rate among the oldest age group and a high risk of infection with an increase in age [1].

The study also found a high prevalence of $S$. mansoni infection in the Southern communities occurring only in the island sites of Tuanikope and Pediatorkope. This could be attributed to the halt of MDA campaigns in these hard-to-reach island communities for almost a decade (as of September 2019) contributing to high infection rates. Furthermore, these communities lack basic social amenities and unavailability of potable water as participants depend solely on water from the river for domestic purposes thus exposing them to infections. Patterns of S. mansoni infection rate showed no significant variation by age and gender since both sexes in these island communities engage in water-related activities which put them at equal risk of infection. The study found a relatively low level of $S$. haematobium infection among participants only in the southern communities. Patterns of $S$. haematobium infection did not differ significantly between communities, gender, and age, owing perhaps to the relatively low levels of infection rate.

The STH infections burden was low in this study with only Hookworm and T. trichiura detected at low levels in the southern communities, but none in the northern community due to the sustained roll out of MDA campaigns in this community (Kpalsogu). Previous studies in Ghana reported prevalence of hookworm infection ranges from 3.2 to $10 \%[17,18]$. The active periodic deworming exercise among school children in some endemic communities in Ghana, targeting intestinal helminths and increased awareness through education, might have been responsible for these low levels of prevalence [13]. The MDA in addition to improved sanitation, access to adequate water supply and proper health care will help significantly, to reduce the burden of STH infection.

The proportion of children with co-infection of parasites in the study was low with children from the island communities (Pediatorkope and Tuanikope) being the most affected. The co-infection of S. mansoni and P. falciparum was the most common. The co-endemicity of these parasites and their vectors coupled with poor sanitary conditions in these communities facilitates transmission of the parasites and a consequent co-infection of these parasites in a single individual. Co-infections of $P$. falciparum and helminths have clinical implications. Previous studies have clearly documented the relationship between intestinal helminth infections, polyparasitism and cognitive functions, growth, and malnutrition among school children. Children with multiple parasitic infections especially those with heavy infection intensity tend to experience more severe cognitive outcomes and other health problems such as malnutrition than children with only one helminth infection $[19,20]$. Studies suggest that co-infection of $P$. falciparum with hookworms or schistosomes tend to exacerbate anaemia and malnutrition morbidities among school children $[15,21]$. The total prevalence of $P$. falciparum and $S$. haematobium co-infection occurred at a very low level. This is consistent with findings from a similar study in Southern Ghana 
which also found a relatively low rate of co-infection of $P$. falciparum and S. haematobium [22]. An important limitation of the current study is the use of only microscopy as a diagnostic method which could also have accounted for the relatively low parasite detection rate as it was possible to miss light infections.

\section{Conclusion}

The study found high burden of single infections of $S$. mansoni and P. falciparum among primary school children living in Southern communities of Ghana. However, parasites co-infection among the children was relatively low recorded only among children from the Southern communities. We recommend expansion of the vector control strategies IRS and LLINs distribution, scaling up of MDA campaigns and provision of basic social amenities to these Southern communities which are hard- toreach to enhance reduction of infection burden in Ghana.

\section{Acknowledgements}

The authors are grateful to the people and school children of Anyakpor, Pediatokorpe, Tuanikorpe and Kpalsogu for permitting us to screen school children in their communities. They greatly appreciate the contribution of Abdul Rahim Mohammed, Benjamin Akuetteh, and all Insecticide Resistance and Malaria Transmission (IMAT) team members for their technical assistance during field surveys and laboratory work. The authors also thank all the community field assistants for helping in the data collection in the various communities.

\section{Authors' contributions}

GAB, SKA, and YAA conceived and designed the study. GAB, KAO, MMO, EAL were responsible for data collection and laboratory processing. YAA and SKA supervised the data collection. GAB, AA and IAH were responsible for the data analysis and drafted the manuscript. All authors read and approved the final manuscript.

\section{Funding}

This study was supported by a grant from the National Institute of Health $(\mathrm{NIH}$ : R01 A1123074 and D43TW011513). The funders had no role or influence on the design of this study, the collection, analysis, and interpretation of the data collected as well as in writing this manuscript.

\section{Availability of data and materials}

All authors confirm that the data supporting the findings of this study are available within the article. All datasets generated and/or analysed during the current study are available from the corresponding author on request. Data request can be made through the corresponding author's email.

\section{Declarations}

\section{Ethics approval and consent to participate}

Ethical approval was provided by the Ethics and Protocol Review Committee (EPRC) of the College of Health Sciences of the University of Ghana (CHS-ET/M.2-4.10/2018-2019). The study was conducted in accordance to the regulations and guidelines of EPRC of the College of Health Sciences of the University of Ghana. The rights and welfare of all participants were protected and the risks involved in this study were considered and minimized. The confidentiality of the data was maintained to ensure the safety and privacy of all participants. Prior to the study, school meetings were held during which the purpose and procedures of the study were discussed. Informed consent was obtained from all subjects and/or their legal guardian(s).

\section{Consent for publication}

Not applicable.

\section{Competing interests}

The authors declare that they have no competing interests.

Received: 3 July 2021 Accepted: 7 December 2021

Published online: 17 December 2021

\section{References}

1. Matangila JR, Doua JY, Linsuke S, Madinga J, Inocêncio Da Luz R, Van Geertruyden JP, et al. Malaria, schistosomiasis and soil-transmitted helminth burden and their correlation with anemia in children attending primary schools in Kinshasa, Democratic Republic of Congo. PLoS ONE. 2014;9(11):1-11.

2. Brooker SJ, Pullan RL, Gitonga CW, Ashton RA, Kolaczinski JH, Kabatereine NB, et al. Plasmodium-helminth coinfection and its sources of heterogeneity across East Africa. J Infect Dis. 2012;205(5):841-52.

3. De Silva NR, Brooker S, Hotez PJ, Montresor A, Engels D, Savioli L. Soiltransmitted helminth infections: updating the global picture. Trends Parasitol. 2003;19(12):547-51.

4. Hotez PJ, Brindley PJ, Bethony JM, King CH, Pearce EJ, Jacobson J. Helminth infections: the great neglected tropical diseases. J Clin Invest. 2008;118(4):1311-21.

5. WHO. Investing to overcome the global impact of neglected tropical diseases: third WHO report on neglected diseases 2015. Geneva: WHO Press, World Health Organization; 2015. p. 2015.

6. Kinung'hi SM, Magnussen P, Kaatano GM, Kishamawe C, Vennervald BJ. Malaria and helminth co-infections in school and preschool children: a cross-sectional study in Magu district, North-Western Tanzania. PLoS ONE. 2014;9(1):1-8.

7. President's Malaria Initiative. Fighting malaria and saving lives: Ghana country profile [Internet]. President's Malaria Initiative. 2020. p. 1-2. Available from: https://www.pmi.gov/docs/default-source/default-docum ent-library/country-profiles/ghana_profile.pdf?sfvrsn=22.

8. Ghana Statistical Service (GSS), ICF. Ghana malaria indicator survey 2019. Accra, Rockville: GSS and ICF; 2019.

9. Yirenya-Tawiah DR, Annang T, Otchere J, Bentum D, Edoh D, Amoah C, et al. Urinary schistosomiasis among adults in the Volta Basin of Ghana: prevalence, knowledge and practices. J Trop Med Parasitol. 2011;34(1):1-16.

10. GHS. Master plan for Neglected Tropical Diseases Programme, Ghana (2016-2020). 2016. p. 31.

11. Ahiadorme M, Morhe E. Soil-transmitted helminth infections in Ghana: a ten-year review. Pan Afr Med J. 2020;35(131):1-16.

12. Lobato L, Miranda A, Faria IM, Bethony JM, Gazzinelli MF. Development of cognitive abilities of children infected with helminths through health education. Rev Soc Bras Med Trop. 2012;45(4):514-9.

13. Ayeh-Kumi PF, Addo-Osafo K, Attah SK, Tetteh-Quarcoo PB, ObengNkrumah N, Awuah-Mensah G, et al. Malaria, helminths and malnutrition: a cross-sectional survey of school children in the South-Tongu district of Ghana. BMC Res Notes. 2016;9(1):1-12.

14. Ayi I, Nonaka D, Adjovu JK, Hanafusa S, Jimba M, Bosompem KM, et al. School-based participatory health education for malaria control in Ghana: engaging children as health messengers. Malar J. 2010;9(1):1-12.

15. Arantza M, Pell C, Manda-Taylor L, Chatio S, Afrah NA, Were F, et al. Prevention and management of malaria during pregnancy: findings from a comparative qualitative study in Ghana, Kenya and Malawi. Malar J. 2013;12(257):1-14.

16. WHO Expert Committee on the Control of Schistosomiasis (2001: Geneva, Switzerland) \& World Health Organization.. Prevention and control of schistosomiasis and soil-transmitted helminthiasis: report of a WHO expert committee. World Health Organization. https://apps.who.int/iris/ handle/10665/42588 Relation. 2002.

17. Magalhães RJS, Biritwum NK, Gyapong JO, Brooker S, Zhang Y, Blair L, et al. Mapping helminth co-infection and co-intensity: geostatistical prediction in Ghana. PLoS Negl Trop Dis. 2011;5(6):1-13.

18. Mirisho R, Neizer ML, Sarfo B. Prevalence of intestinal helminths infestation in children attending Princess Marie Louise Children's Hospital in Accra, Ghana. J Parasitol Res. 2017;2017:1-8. 
19. Mwangi TW, Bethony JM, Brooker S. Malaria and helminth interactions in humans: an epidemiological viewpoint. Ann Trop Med Parasitol. 2007;100(7):551-70.

20. Jardim-Botelho A, Raff S, De Ávila RR, Hoffman HJ, Diemert DJ, CorrêaOliveira R, et al. Hookworm, Ascaris lumbricoides infection and polyparasitism associated with poor cognitive performance in Brazilian schoolchildren. Trop Med Int Health. 2008:13(8):994-1004.

21. Shapiro AE, Tukahebwa EM, Kasten J, Clarke SE, Magnussen P, Olsen $A$, et al. Epidemiology of helminth infections and their relationship to clinical malaria in southwest Uganda. Trans R Soc Trop Med Hyg. 2005;99(1):18-24.

22. Nyarko R, Torpey K, Ankomah A. Schistosoma haematobium, Plasmodium falciparum infection and anaemia in children in Accra, Ghana. Trop Dis Travel Med Vaccines. 2018;4(1):1-6.

\section{Publisher's Note}

Springer Nature remains neutral with regard to jurisdictional claims in published maps and institutional affiliations.

- fast, convenient online submission

- thorough peer review by experienced researchers in your field

- rapid publication on acceptance

- support for research data, including large and complex data types

- gold Open Access which fosters wider collaboration and increased citations

- maximum visibility for your research: over $100 \mathrm{M}$ website views per year

At $\mathrm{BMC}$, research is always in progress.

Learn more biomedcentral.com/submissions 